\title{
INDUCTIVE-RESONANT THEORY OF NONRADIATIVE TRANSITIONS FOR COMPLEX ORGANIC MOLECULES IN CONDENSED PHASE*
}

\author{
V.L. ERMolaev ${ }^{\dagger}$ and E.B. Sveshnikova \\ S.I. Vavilov State Optical Institute, Birzhevaya liniya 12, 199034 St. Petersburg, Russia
}

(Received July 23, 1998; revised version October 20, 1998)

\begin{abstract}
Ours and literature data on influence of replacement of deuterium by protium in complex organic molecules on nonradiative triplet-singlet transition rates are discussed from the standpoint of the inductive-resonant theory of this process. It has been shown that the extent to which the protium introduction in molecule affects the nonradiative rate depends on distance between protium atom and the vibronic molecular oscillator of triplet-singlet transition and on $\pi$-electronic density near-by protium.
\end{abstract}

PACS numbers: $33.50 . \mathrm{Dq}, 78.30 . \mathrm{Jw}$

\section{Introduction}

Over the course of the past 70 years, the mechanism of nonradiative transitions between electronic levels of complex organic molecules in condensed phase was the most intriguing problem for those interested in luminescence, spectroscopy, photochemistry, and photobiophysics. As Prof. S.I. Vavilov wrote: "The most difficult thing is not so much to explain luminescence in the condensed phase as, vice versa, to account for the lack of luminescence in so many molecules" [1]. In spite of the great progress in this field during the last 30 years the scientists are still far from final decision of the problem.

Nonradiative deactivation of electronic excitation can take place by means of physical or chemical mechanisms. Under the physical mechanism, the only result of deactivation of a molecular electronic excitation is the transition to a lower-energy electronic state and the transformation of the released energy first to vibrational energy and then to thermal one. Deactivation by the chemical mechanism results in final or intermediate chemical products differing from the initial compound.

*The results of this paper were initially presented at The Jabfonski Centennial Conference on Luminescence and Photophysics, July 23-27, 1998, Toruń, Poland.

† e-mail: ermol@soi.spb.su

$\ddagger$ e-mail: svesh@soi.spb.su 
Typical examples of chemical deactivation mechanisms are photoisomerization and electron transfer. In this paper we shall discuss a physical mechanism only.

There are both radiative and nonradiative transitions between three levels of the Jabłoński Diagram for complex organic molecules [2,3]. It was shown in the paper of one of the authors and of Lubimtzev in the proceedings devoted to Fifty Years of the Jabłoński Diagram [4] that nonradiative transitions between high excited levels of complex molecules occur at extremely high rates $k_{n \mathrm{n}}\left(S_{n}-S_{n-1}\right)=$ $10^{12}-10^{14} \mathrm{~s}^{-1}$. In the given report we shall consider, on the contrary, mechanism of the slowest nonradiative transitions from the lowest metastable triplet levels to the ground levels. The rates of this process in the luminescent molecules $k_{\mathrm{nr}}\left(T_{1}-S_{0}\right)$ are $\left(10^{5}-10^{-2} \mathrm{~s}^{-1}\right)$.

The mechanism of nonradiative transitions has been considered in a few monographs $[5,6]$ and in some chapters of different books on spectroscopy, luminescence, and photochemistry [7-9].

We try to show the possibility of the inductive-resonant theory of nonradiative transitions to describe the regularities of such processes. At first the validity of the theory was proved by us for nonradiative transitions of lanthanide ions in solutions [10]. Subsequently it was proved for transition metal ions and then it was extended with the clauses to complex organic molecules [11]. Now we shall return to the last mentioned problem and shall put forward additional arguments in favor of this expansion.

Most of the theories of nonradiative transitions takes the operator of nonadiabaticity $(\partial H / \partial Q)$ as a perturbation which induces the nonradiative transition. In some papers spin-orbit operator $\left(H_{\mathrm{so}}\right)$ is taken as such perturbation for the cases of intersystem crossing. Henry and Siebrand [12] suppose that for intersystem crossing in molecules the sum of these two operators must be taken into consideration in the general case. They suppose also that for different molecules nonadiabaticity operator or spin-orbit operator may predominantly define the magnitude of matrix element for intersystem crossing. The authors of Refs. [11-13] pointed out that the consideration of only vibrational part of matrix element of nonradiative transitions allows one to account for the effect of full deuteration of molecules on $k_{\mathrm{nr}}$, but such consideration cannot explain the $k_{\mathrm{nr}}$ dependence on position of atoms substituted in molecules and cannot explain the difference in $k_{\mathrm{nr}}$ magnitude for transitions from triplet sublevels under low temperatures. Henry and Siebrand [12] are of the opinion that only the inclusion of $H_{\text {so }}$ operator and consideration of electronic term consisting of one-center integrals allows one to explain the locality of effects because the largest matrix element is encountered when the highest local $\pi$-electron density coincides with the largest local out-of-plane amplitude of $\mathrm{C}-\mathrm{H}$ vibration.

In this paper we suggest alternative explanation of substitutent effect. The inductive-resonant theory of nonradiative transitions [13] predicts all nonradiative transition regularities and $k_{\mathrm{nr}}$ magnitudes in themselves for lanthanide and transition metal ions in condensed matter and a number of regularities for organic molecules $[11,13]$. Under some assumptions the inductive-resonant theory is able to account for the difference in the influence of substitutents on $k_{\mathrm{nr}}$ for $T_{1}-S_{0}$ transitions as will be shown below. 
The assumptions used by the inductive-resonant theory are:

(1) In the most of organic molecules vibrations may be divided into two separate groups: high-frequency strongly anharmonic stretching vibrations, such as $\mathrm{C}-\mathrm{H}, \mathrm{C}-\mathrm{D}, \mathrm{N}-\mathrm{H}, \mathrm{N}-\mathrm{D}, \mathrm{O}-\mathrm{H}, \mathrm{O}-\mathrm{D}$, which form a vibrational spectrum of molecules in the overtone region, the spectrum which overlaps the phosphorescence spectrum of molecule and vibrations forming vibronic spectrum of molecules, such as $\mathrm{C}-\mathrm{C}$, $\mathrm{C}=\mathrm{O}, \mathrm{C}-\mathrm{N}$. This assumption allows us to calculate a vibrational matrix element through an integral of overlapping of phosphorescence spectrum and vibrational spectrum of molecules. Such approach allows us to take into account simultaneously displacement and distortion of adiabatic potential under excitation for vibrations forming phosphorescence spectrum and anharmonicity of high-frequency vibrations.

(2) The existence of Coulombic interaction between vibronic oscillator of phosphorescent transition and high-frequency vibrational oscillator is supposed and as a result of it, it is also supposed that for each atom of molecule the interaction of vibronic oscillator localized on it is the most for the high-frequency vibrations nearest to it and it is the most also if local electronic density is the highest for this atom.

(3) A small displacement of H-atom under its vibrations and a small displacement of electronic density under excitation in comparison with the distance between $\mathrm{C}$ and $\mathrm{H}$-atoms allow us to make the expansion of their Coulombic interaction in a power series in multipoles and to restrict our consideration to dipole-dipole approximation. Thus we obtain Förster formula for interaction of vibronic and vibrational oscillator.

In accordance with such approach the nonradiative transitions in molecules occur due to interaction with high-frequency stretching vibrations (but not with out-of-plane) and these vibrations take the marked part of excitation energy and therefore, it is reasonable to name them promoting-accepting vibrations and another type of vibrations which form vibronic spectrum and take a certain part of energy to name accepting vibrations. In condensed phase, the surplus of vibrational energy dissipates rapidly in the environment [14]. The suggested scheme is given in Fig. 1.

Such approach may be proved and based quantum mechanically on usual consideration of the process. The computation of $k_{\mathrm{nr}}$ in many systems is based on the following assumptions: (a) The adiabatic approximation is correct; (b) Adiabatic wave functions are not eigenfunctions of the total Hamiltonian $H$ of the system. Therefore, the nonadiabatic operator $V$ is responsible for nonradiative transitions between different adiabatic electronic states. This operator has only nondiagonal (in electronic quantum numbers) nonze;o matrix elements; (c) Interferential effects between different channels of nonradiative transitions are neglected. This allows the total rate $k_{\mathrm{nr}}$ of transitions between triplet and ground states to be written as a sum of partial transition probabilities; (d) It is supposed that the excited electronic state is characterised by the equilibrium distribution over vibrational sublevels during the entire period of nonradiative transition. This is possible if the vibrational relaxation rate significantly exceeds that of the nonradiative $T_{1}-S_{0}$ transition; (e) The vibrational wave function is represented as 


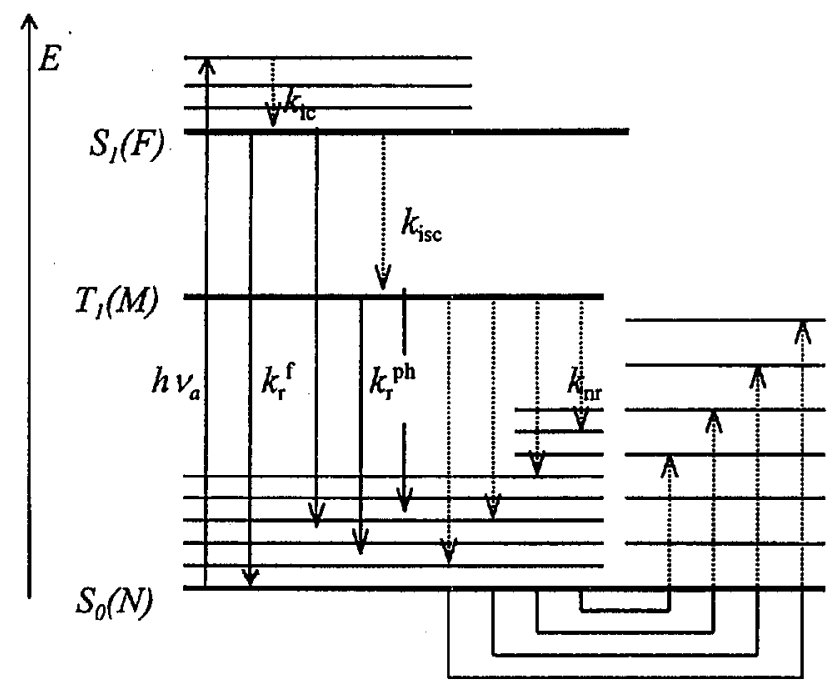

Fig. 1. Diagram of levels for illustration of the inductive-resonant theory of nonradiative transitions from the lowest excited triplet level of complex organic molecules. Bold horizontal lines are electronic levels, thin horizontal lines are vibronic and vibrational levels. The left hand side of the scheme represents vibronic levels of molecule excited under electronic transition (e.g. as a rule $\mathrm{C}-\mathrm{C}$ skeletal vibrations, $\mathrm{C}=\mathrm{O}$ valent vibrations, etc. named by us as accepting vibrations). The right hand side shows levels of the highest-frequency strong anharmonic vibrations of the same molecule (e.g. C-H(D), $\mathrm{O}-\mathrm{H}(\mathrm{D}), \mathrm{N}-\mathrm{H}(\mathrm{D})$ stretching vibrations) which we call promoting-accepting vibrations. Vertical dashed lines denote electronic-vibrational inductive-resonant (Förster's type) energy transfer responsible for the nonradiative transitions. Excess vibrational energy of molecule dissipates fast in vibrational relaxation [14].

the product of wave functions of normal vibrations. These assumptions should be supplemented with the formulated above division of vibrations into two groups: promoting-accepting and accepting. Under such assumption Franck-Condon term may be written as a usual overlapping integral of phosphorescence spectrum and vibrational absorption and $k_{\mathrm{nr}}$ can be written as

$$
k_{\mathrm{nr}} \sim\left|\left\langle\varphi_{0}\left|\overline{\frac{\partial U(r, Q)}{\partial Q_{k}}}\right| \varphi_{1}\right\rangle\right|^{2} \int f(E) \sigma(E) E^{-(s+1)} \mathrm{d} E,
$$

where $U(r, Q)$ is the Coulomb energy of electron-nucleus interaction, $Q_{k}$ is nuclear coordinates of promoting-accepting vibrations, the bar denotes averaging over promoting-accepting vibrations, $f(E)$ is the normalised electronic-vibrational phosphorescence spectrum of molecule, $\sigma(E)$ is the cross-section of vibrational absorption of promoting-accepting vibrations in the same molecule.

Under calculation of the electronic matrix element $\left\langle\varphi_{0}\left|\partial U / \partial Q_{k}\right| \varphi_{1}\right\rangle$, one may confine oneself to the dipole approximation

$$
k_{\mathrm{n} \dot{r}}=\frac{9000 \ln (10) \kappa^{2} k_{\mathrm{r}}}{128 \pi^{5} n^{4} N_{\mathrm{A}} R^{6}} \int I_{\mathrm{ph}}^{n}(\nu) \varepsilon_{\mathrm{vib}}(\nu) \nu^{-4} \mathrm{~d} \nu .
$$


Here, $k_{\mathrm{r}}$ is the radiative transition rate of phosphorescence in electric dipole approximation, $n$ is the refractive index of the medium, $\nu$ is the wave numbers, $N_{\mathrm{A}}$ is Avogadro number, $R$ is the effective distance between the vibronic and promoting-accepting vibrational oscillators, $I_{\mathrm{ph}}^{n}(\nu)$ is the phosphorescence spectrum normalised to a unit area, $\varepsilon_{\mathrm{vib}}(\nu)$ is the molar decimal extinction coefficient of promoting-accepting vibrations, $\kappa^{2}$ is the orientation factor.

\section{Results and discussion}

A number of consequences follows from the inductive-resonant theory of nonradiative transitions:

(1) The value of $k_{\mathrm{nr}}$ for $T_{1}-S_{0}$ transition of different molecules should be directly proportional to the integral of overlapping of a phosphorescence spectrum normalized to the unit area and a vibrational spectrum of absorption of promoting-accepting vibrations, provided: $k_{\mathrm{r}}, \kappa^{2}$ and a distance between $T_{1}-S_{0}$ transition oscillator and groups, responsible for promoting-accepting vibrations, do not vary. An increase in this distance results in reduction of $k_{\mathrm{nr}}$. At an identical height of a triplet level, $k_{\text {nr }}$ will be greater, when the phosphorescence spectrum is wider (slower decrease towards small frequencies). Hence it follows that the influence of deuteration of the molecule on $k_{\mathrm{nr}}$ is larger when a phosphorescence spectrum is located at large frequencies (do not confuse with the deuteration influence on $\tau_{\mathrm{ph}}$, which is the greatest when $T_{1}$ level is located at $20000 \mathrm{~cm}^{-1}$ ).

(2) If the multiple expansion of interaction operator is invoked and dipoledipole approximation is true, the proportionality of $k_{\mathrm{nr}}$ and rate constant of appropriate radiative transition $\left(k_{\mathrm{r}}\right)$ should be expected. Indeed, the experimental facts confirm this assumption. Examination of halogennaphthalene derivatives which have approximately identical energy of triplet level gives an example of validity of such prediction. Indeed, $k_{\mathrm{r}}$ is enhanced $\sim 200$ times from 1-chloronaphthalene to 1-iodonaphthalene, and simultaneously $k_{\mathrm{nr}}$ is increased approximately in the same manner. Additional evidence of $k_{\mathrm{r}}$ and $k_{\mathrm{nr}}$ proportionality provides analysis of temperature dependence of $T_{1}-S_{0}$ transition rates for $\mathrm{Ru}(\mathrm{bpy})_{3}^{2+}$ complexes in ethanol solution. It is shown that in the range of 2-77 $\mathrm{K}$ phosphorescence decay is varied from 230 up to $6 \mu \mathrm{s}$, but phosphorescence yield is not changed. The deuteration of complex results in an identical variation of $\tau_{\mathrm{ph}}$ and phosphorescence yield which points out that in this system $k_{\mathrm{nr}}$ and $k_{\mathrm{r}}$ are varying in exactly parallel manner [15]. Data on rate constants of radiative and nonradiative transitions from individual sublevels of triplet states of a number of molecules collected in paper [13] also demonstrate the approximate parallelism of $k_{\mathrm{nr}}$ and $k_{\mathrm{r}}$. The exact proportionality of $k_{\mathrm{nr}}$ and $k_{\mathrm{r}}$ cannot be achieved for the last case because radiative transitions from individual sublevels of triplet state have different orientations about molecule and as a result, have different interactions with promoting-accepting vibrations. The idea of proportionality between a rate constant of nonradiative $T_{1}-S_{0}$ transition in a molecule and a rate constant competing with its radiative transition is not pertinent only to our works $[7,11,13]$ but can also be found in other articles $[16,17]$.

Thus though the direct comparison of the sizes of an electronic shell, vibrating groups of atoms and distances between them does not permit an expansion of 
Coulombic interaction of electrons and nuclea in terms of multipoles, the observed proportionality $k_{\mathrm{nr}}$ and $k_{\mathrm{r}}$ is an argument in favor of applicability of d-d approach to these systems. If it is possible we must compare the distance between electronic shell and promoting-accepting group with the amplitude of displacement of electronic shell and light atom of promoting-accepting group, i.e., with essentially smaller sizes.

The useful information on localization of vibronic transition and $k_{\mathrm{nr}}$ dependence on distance between vibronic and vibrational oscillators is obtained from analysis of contribution of various promoting-accepting groups of molecule in $k_{\mathrm{nr}}$ value. The information is obtained from comparison of influence of substitution of deuterium for protium in various positions of molecules on phosphorescence

\section{TABLE I}

The influence of replacement of deuterium by protium in substituted naphthalenes on rate constants of nonradiative $T_{1}-S_{0}$ transitions, in ethanol and ethanol- $d_{6}, 77 \mathrm{~K}[18,19]$.

\begin{tabular}{|c|c|c|c|c|}
\hline Compound & $\begin{array}{c}\tau_{\mathrm{ph}} \\
{[\mathrm{s}]}\end{array}$ & $\begin{array}{c}k_{\mathrm{r}}+k_{\mathrm{nr}} \\
{\left[\mathrm{s}^{-1}\right]}\end{array}$ & $\begin{array}{c}k_{\mathrm{nr}}^{\prime}{ }^{a}(\mathrm{H}) \\
{\left[\mathrm{s}^{-1}\right]}\end{array}$ & $\begin{array}{l}k_{\mathrm{nr}}^{\prime} \\
{\left[\mathrm{s}^{-1}\right]}\end{array}$ \\
\hline $1-\mathrm{D}_{2}-\mathrm{Naph}$ thylamine- $d_{7}^{\mathrm{c}}$ & 11.0 & 0.091 & - & - \\
\hline $1-\mathrm{H}_{2}-\mathrm{Naph}$ thylamine- $d_{7}$ & 2.7 & 0.37 & 0.14 & \\
\hline $1-\mathrm{H}_{2}-\mathrm{Naphthylamine}-h_{7}$ & 1.0 & 1.0 & 0.14 & .0 .09 \\
\hline $1,5-\mathrm{D}_{4}$-Naphthylenediamine- $h_{6}$ & 1.55 & .0 .65 & & \\
\hline $1,5-\mathrm{H}_{4}-\mathrm{Naph}$ thylenediamine- $h_{6}$ & 0.75 & 1.33 & 0.17 & - \\
\hline $1-\mathrm{D}_{3}-\mathrm{Naphtylammonium-} d_{7}$ & 10.0 & 0.1 & & \\
\hline $1-\mathrm{H}_{3}-\mathrm{Naph}$ tylammonium- $d_{7}$ & 2.5 & 0.4 & 0.1 & \\
\hline $1-\mathrm{H}_{3}-\mathrm{Naph}$ tylammonium- $h_{7}$ & 1.25 & 0.8 & 0.1 & 0.06 \\
\hline $1-\mathrm{D}_{3}$-Naphtylammonium- $h_{7}$ & 1.7 & 0.59 & 0.07 & 0.07 \\
\hline 2-D-Naphthol- $d_{7}$ & 11.0 & 0.09 & & \\
\hline 2-H-Naphthol- $d_{7}$ & 4.7 & 0.21 & 0.12 & \\
\hline 2-D-Naphthol- $h_{7}$ & 1.4 & 0.71 & & 0.09 \\
\hline 2-H-Naphthol- $h_{7}$ & 1.3 & 0.77 & 0.06 & \\
\hline 2-D-Naphthaldehyde- $d_{7}$ & 4.8 & 0.21 & & \\
\hline 2-D-Naphthaldehyde- $h_{7}$ & 1.35 & 0.74 & & 0.08 \\
\hline 2-H-Naphthaldehyde- $d_{7}$ & 0.46 & 2.17 & 1.96 & \\
\hline 2-H-Naphthaldehyde- $h_{7}$ & 0.37 & 2.70 & & 0.08 \\
\hline \multicolumn{5}{|c|}{$\begin{array}{l}{ }^{a} k_{\mathrm{nr}}^{\prime} \text { is the contribution to } k_{\mathrm{nr}} \text { from replacement of one } \mathrm{D} \text { by } \mathrm{H} \\
\text { atom in naphthalene substituents. } \\
{ }^{b} \text { The same for replacement in a naphthalene ring. } \\
{ }^{c} \mathrm{D} \text { and } \mathrm{H} \text { capital letters refer to substituent atoms, } d \text { and } h-\text { to } \\
\text { naphthalene ring. }\end{array}$} \\
\hline
\end{tabular}


TABLE II

The contribution from replacement of deuterium by protium in different places of biphenyl (B) molecule to the rate constants of $T_{1} \rightarrow S_{0}$ nonradiative transitions (solutions in EPA at $77 \mathrm{~K}[20]$ ).

\begin{tabular}{|c|c|c|c|c|c|c|}
\hline Compound & $\begin{array}{c}\tau_{\mathrm{ph}} \\
{[\mathrm{s}]} \\
\end{array}$ & $\begin{array}{c}k_{\mathrm{r}}+k_{\mathrm{n} \boldsymbol{r}} \\
{\left[\mathrm{s}^{-1}\right]}\end{array}$ & $\begin{array}{c}\sum k_{\mathbf{n}_{\mathrm{r}}}^{\prime}(\mathrm{H}) \\
{\left[\mathrm{s}^{-1}\right]}\end{array}$ & $\begin{array}{c}k_{\mathrm{nr}}^{\prime} \\
{\left[\mathrm{s}^{-1}\right]}\end{array}$ & Place & $\sum_{\text {(calc.) }} k_{\mathrm{nr}}^{\prime}$ \\
\hline Biphenyl- $h_{0}$ & 10.3 & 0.097 & 0.00 & & & \\
\hline $4,4^{\prime}-\mathrm{B}-h_{2}$ & 7.2 & 0.139 & 0.042 & 0.021 & $4\left(4^{\prime}\right)$ & 0.042 \\
\hline $3,3^{\prime}, 5,5^{\prime}-\mathrm{B}-h_{4}$ & 8.7 & 0.115 & 0.018 & 0.005 & $3,5\left(3^{\prime}, 5^{\prime}\right)$ & 0.020 \\
\hline $3,3^{\prime}, 4,4^{\prime}, 5,5^{\prime}-\mathrm{B}-h_{6}$ & 6.4 & 0.156 & 0.059 & $\begin{array}{l}0.021 \\
0.005\end{array}$ & $\begin{array}{l}4\left(4^{\prime}\right) \\
3,5\left(3^{\prime}, 5^{\prime}\right)\end{array}$ & 0.062 \\
\hline $2,2^{\prime}, 4,4^{\prime}, 6,6^{\prime}-\mathrm{B}-h_{6}$ & 5.2 & 0.192 & 0.095 & $\begin{array}{l}0.021 \\
0.013\end{array}$ & $\begin{array}{l}4\left(4^{\prime}\right) \\
2,6\left(2^{\prime}, 6^{\prime}\right)\end{array}$ & 0.094 \\
\hline $2,2^{\prime}, 3,3^{\prime}, 5,5^{\prime}, 6,6^{\prime}-\mathrm{B}-h_{8} \quad\left(\mathrm{~B}-d_{2}\right)$ & 5.7 & 0.175 & 0.078 & $\begin{array}{l}0.013 \\
0.005\end{array}$ & $\begin{array}{l}2,6\left(2^{\prime}, 6^{\prime}\right) \\
3,5\left(3^{\prime}, 5^{\prime}\right)\end{array}$ & 0.072 \\
\hline Biphenyl- $h_{10}$ & 4.3 & 0.233 & 0.136 & $\begin{array}{l}0.021 \\
0.013 \\
0.005\end{array}$ & $\begin{array}{l}4\left(4^{\prime}\right) \\
2,6\left(2^{\prime}, 6^{\prime}\right) \\
3,5\left(3^{\prime}, 5^{\prime}\right)\end{array}$ & 0.114 \\
\hline
\end{tabular}

decay. Tables I, II and III provide data on the contribution of different protonated groups in naphthalene, biphenyl and anthracene derivatives to the rate constants of $T_{1}-S_{0}$ nonradiative transitions [18-25]. We have tried to estimate the change of magnitude of a rate constant of nonradiative $T_{1}-S_{0}$ transition ( $\left.k_{\mathrm{nr}}^{\prime}\right)$ at replacement in a molecule of a separate deuterium atom by protium atom. The naphthalene and biphenyl derivatives were selected because their phosphorescence decay depends strongly on replacement of a deuterium by protium. Table I summarises the $k_{\mathrm{nr}}^{\prime}$ rate at replacement of $\mathrm{D}$ - by $\mathrm{H}$-atom in naphthalene ring with $k_{\mathrm{nr}}^{\prime}$ at replacement in naphthalene substituting group ( $\mathrm{NH}_{2}, \mathrm{OH}$ and $\mathrm{CHO}$ (aldehyde group)). Under examination of the data exhibited in Table I we notice that the protium introduction into the above-mentioned substituents stronger influences the $k_{\mathrm{nr}}$ rate constant than the introduction of $\mathrm{H}$ into naphthalene ring. The special attention attracts the very strong influence of the replacement of $\mathrm{D}$ by $\mathrm{H}$ in aldehyde group of naphthaldehyde on the $k_{\mathrm{nr}}$. And these results are not surprising from the position of the inductive-resonant theory. It is necessary to notice that the introduction of aldehyde group in the naphthalene ring weakly influences the position and structure of phosphorescence spectrum. Mutual analysis of 1-naphthylamine and 1-naphthylammonium phosphorescence spectra and their $\mathrm{N}-\mathrm{H}(\mathrm{D})$ groups influence on $k_{\mathrm{nr}}$ shows that the decrease in $\mathrm{NH}$-groups participation in $\pi$-conjugation $\left(\mathrm{NH}_{3}\right.$ group compared with $\mathrm{NH}_{2}$ ) diminishes their contribution to $k_{\mathrm{nr}}$. And these results are not surprising from the position of the inductive-resonant theory because $\mathrm{N}$ and $\mathrm{O}$ atoms take an active part in $\pi$-conjugation and $\mathrm{N}-\mathrm{H}$ and $\mathrm{O}-\mathrm{H}$ groups cannot be considered as groups remote from the region of vibronic transition localization. 
TABLE III

The phosphorescence decay of partially deuterated naphthalenes $(N)$ and anthracenes $(\mathrm{A})$ at $77 \mathrm{~K}$ and the contribution in $k_{\mathrm{nr}}$ from separate $\mathrm{C}-\mathrm{H}$ bond $\left(k_{n r}^{\prime}\right)$.

\begin{tabular}{|c|c|c|c|c|c|c|c|}
\hline \multicolumn{2}{|l|}{ Compounds } & \multirow{2}{*}{$\begin{array}{c}\tau_{\mathrm{ph}}{ }^{a} \\
{[\mathrm{~s}]}\end{array}$} & \multirow{2}{*}{$\begin{array}{c}\tau_{\mathrm{ph}}{ }^{b} \\
{[\mathrm{~s}]}\end{array}$} & \multirow{2}{*}{$\begin{array}{c}\begin{array}{c}\tau_{\mathrm{ph}}{ }^{c} \\
{[\mathrm{~s}]}\end{array} \\
16.2\end{array}$} & \multirow{2}{*}{$\begin{array}{l}k_{\mathrm{n} r}^{\prime a} \\
{\left[\mathrm{~s}^{-1}\right]}\end{array}$} & \multirow{2}{*}{$\begin{array}{l}k_{\mathrm{nr}}^{\prime b} \\
{\left[\mathrm{~s}^{-1}\right]}\end{array}$} & \multirow{2}{*}{$\begin{array}{l}k_{\mathrm{nr}}^{\prime}{ }^{c} \\
{\left[\mathrm{~s}^{-1}\right]}\end{array}$} \\
\hline Naphthalene- $h_{0}$ & $\left(\mathrm{~N}-d_{8}\right)$ & & & & & & \\
\hline $1-N-h_{1}$ & $\left(\mathrm{~N}-d_{7}\right)$ & 11.08 & 11.07 & & 0.044 & 0.045 & \\
\hline $2-\mathrm{N}-h_{1}$ & $\left(\mathrm{~N}-d_{7}\right)$ & 13.51 & 13.35 & & 0.028 & 0.029 & \\
\hline $1,4,5,8-\mathrm{N}-h_{4}$ & $\left(\mathrm{~N}-d_{4}\right)$ & & & 4.8 & & & 0.036 \\
\hline $2,3,5,6-\mathrm{N}-h_{4}$ & $\left(\mathrm{~N}-d_{4}\right)$ & & & 5.4 & & & 0.030 \\
\hline $1,4,5,6,7,8-\mathrm{N}-h_{6}$ & $\left(\mathrm{~N}-d_{2}\right)$ & 3.15 & 3.15 & & 0.045 & 0.045 & \\
\hline $1,3,4,5,6,8-\mathrm{N}-h_{6}$ & $\left(\mathrm{~N}-d_{2}\right)$ & 3.12 & 3.17 & & 0.046 & 0.045 & \\
\hline $2,3,5,6,7,8-\mathrm{N}-h_{6}$ & $\left(\mathrm{~N}-d_{2}\right)$ & 3.64 & 3.66 & 3.4 & 0.038 & 0.038 & 0.038 \\
\hline $2,3,4,6,7,8-\mathrm{N}-h_{6}$ & $\left(N-d_{2}\right)$ & 3.70 & 3.69 & & 0.037 & 0.038 & \\
\hline $2,3,4,5,6,7-\mathrm{N}-h_{6}$ & $\left(\mathrm{~N}-d_{2}\right)$ & 3.70 & 3.72 & & 0.037 & 0.037 & \\
\hline $1,3,4,5,6,7,8-\mathrm{N}-h_{7}$ & $\left(N-d_{1}\right)$ & 2.75 & 2.85 & 2.6 & 0.045 & 0.044 & 0.046 \\
\hline $2,3,4,5,6,7,8-\mathrm{N}-h_{7}$ & $\left(N-d_{1}\right)$ & 2.94 & 3.03 & 2.8 & 0.042 & 0.041 & 0.042 \\
\hline \multirow[t]{2}{*}{ Naphthalene- $h_{8}$} & $\left(N-d_{0}\right)$ & 2.51 & 2.60 & 2.5 & 0.044 & 0.042 & 0.042 \\
\hline & & $\begin{array}{l}\tau_{\mathrm{ph}}{ }^{d} \\
{[\mathrm{~s}]}\end{array}$ & $\begin{array}{l}\tau_{\mathrm{ph}}{ }^{2} \\
{[\mathrm{~s}]}\end{array}$ & & $\begin{array}{l}k_{\mathrm{nr}}^{\prime d} \\
{\left[\mathrm{~s}^{-1}\right]}\end{array}$ & $\begin{array}{l}k_{\mathrm{nr}}^{\prime}{ }^{e} \\
{\left[\mathrm{~s}^{-1}\right]}\end{array}$ & \\
\hline Anthracene- $h_{0}$ & $\left(\mathrm{~A}-d_{10}\right)$ & 0.112 & 0.117 & & & & \\
\hline $9,10-\mathrm{A}-h_{2}$ & $\left(A-d_{8}\right)$ & 0.075 & & & 2.2 & & \\
\hline $1,2,3,4,9,10-\mathrm{A}-h_{6}$ & $\left(\mathrm{~A}-d_{4}\right)$ & & 0.047 & & & 2.12 & \\
\hline $1,2,3,4,5,6,7,8-\mathrm{A}-h_{8}$ & $\left(\mathrm{~A}-d_{2}\right)$ & & 0.052 & & & 1.34 & \\
\hline Anthracene- $h_{10}$ & $\left(A-d_{0}\right)$ & 0.040 & 0.036 & & 1.6 & 1.92 & \\
\hline
\end{tabular}

Table II gives the $k_{n r}^{\prime}$ values at $\mathrm{H}$ introduction in different positions of biphenyl molecule. The $k_{\mathrm{nr}}^{\prime}$ values for different positions in biphenyl molecule are arranged in the following way. The greatest value has $k_{\mathrm{nr}}^{\prime}$ for $4,4^{\prime}$ positions. The lesser value has $k_{\mathrm{nr}}^{\prime}$ for $2,2^{\prime}, 6,6^{\prime}$ positions and the least value has $k_{\mathrm{nr}}^{\prime}$ for $3,3^{\prime}, 5,5^{\prime}$ positions. The ratio between the magnitude of $k_{\mathrm{nr}}^{\prime}$ of $4,4^{\prime}$ and $3,3^{\prime}, 5,5^{\prime}$ positions is equal to four.

The results of investigation of influence of replacement of D-atom with $\mathrm{H}$-atom in different positions of naphthalene and anthracene rings are collected in Table III. Again there is the difference of substitution in different positions of these molecules. The strongest effect is observed for $\mathrm{H}$ in 1,4,5,8 positions of naphthalene ring and in 9,10 positions of anthracene ring. The distinctions are less than for biphenyl but reasonably determinable. The $1,4,5,8$ positions in naphthalene and 9,10 positions in anthracene have the greatest chemical activity. There is the correlation between influence of H-substitution on $k_{\mathrm{nr}}$ and NMR chemical shift 
of proton in this position. The values of NMR chemical proton shift $(\delta$, millionth part) are in naphthalene: position $1-7.81$, position $2-7.46$; in anthracene: position $9-8.31$, position $1-7.91$ and position $2-7.39$ [26].

\section{Conclusion}

As was mentioned before, consideration of vibrational matrix element of transition allows us to account for the total deuteration effect but cannot explain the affect of position of substituting groups on this effect and the difference of $k_{\mathrm{nr}}$ magnitudes for sublevels of triplet states. These two facts are explained by both Henry-Siebrand and inductive-resonant theories, therefore, both interpretations use partly the similar assumptions. Henry and Siebrand take $H_{\text {so }}$ as the main perturbating factor, the inductive-resonant theory uses the first term of expansion in power series of the operator of electron-phonon interaction and is confined oneself to the dipole approximation. According to the last theory, spin-orbit interaction is taken into account in electronic matrix element and it leads to a direct proportionality of $k_{\mathrm{nr}}$ and $k_{\mathrm{r}}$ in those systems where the change of spin-orbit interaction does not lead to the change of vibrational matrix element. Thus both theories may account for the difference in $k_{\mathrm{nr}}$ for transitions from different sublevels of triplet states. In order to account for the position-dependent deuterium effect, both theories take into account the electronic density distribution in molecules, but as before in Ref. [12], $H_{\text {so }}$ is considered as the main operator, responsible for the combining of the $\pi$-electronic cloud with $\mathrm{CH}$-out-of-plane bending vibrations and on this basis substitution effect is accounted. The inductive-resonant theory also takes into account the electronic density distribution in molecules, the participation of substitutent groups in $\pi$-conjugation, but as before it considers the interaction of electronic or more precisely vibronic oscillator with $\mathrm{CH}, \mathrm{NH}, \mathrm{OH}$-high-frequency stretching vibrations. It ought to note that the interaction just with these vibrations defines the magnitude of $k_{\mathrm{nr}}$ for metal ions in solutions. Both theories predict that the increase in $\pi$-electronic density on $\mathrm{C}, \mathrm{N}$ and $\mathrm{O}$-atoms leads to the increase in the participation of this local center in nonradiative transition. The prediction of the inductive-resonant theory is based on the fact that the parameter $R$ (the distance between vibronic oscillator and oscillator of local vibration) depends on electronic density distribution and mutual orientation of oscillators. The participation of substituting groups such as $\mathrm{NH}, \mathrm{OH}$ in $\pi$-conjugation leads to the diminution of distance between oscillators of vibronic and vibrational ( $\mathrm{NH}, \mathrm{OH}$ ) transitions.

As a summary it should be pointed out that there is no necessity to consider several interaction operators for description of all data for nonradiative transitions of different molecules and metal ions but the possibility exists to confine oneself to the consideration of only inductive-resonant interaction of vibronic and vibrational oscillators.

Inasmuch as replacement of deuterium by protium and introduction of substituents have no marked influence on the phosphorescence spectrum position but have strong influence on nonradiative rate of $T_{1}-S_{0}$ transition, it is evident that only taking into account the direction of transition moment of vibronic and vibrational transitions as well as the conjugation of substituting group with ring 
allows us to explain all data for partially protonated molecules. Thus these data confirm the necessity of taking into account the factors of the distance and mutual orientation between vibronic and vibrational oscillators that correlates with inductive-resonant theory of nonradiative transitions.

The authors gratefully acknowledge financial support from Russian Fund of Basic Investigations grant No. 97-03-32398.

\section{References}

[1] S.I. Vavilov, Bull. Acad. Sci. USSR, Ser. Phys. 9, 283 (1945).

[2] A. Jabłoński, Nature 131, 839 (1933).

[3] A. Jabłonski, Z. Phys. 94, 38 (1935).

[4] V.L. Ermolaev, V.A. Lubimtzev, Acta Phys. Pol. A 71, 731 (1987).

[5] R. Englman, Non-Radiative Decay of Ions and Molecules in Solids, North Holland, Amsterdam 1979, p. 336.

[6] E.S. Medvedev, V.I. Osherov, Radiationless Transitions in Polyatomic Molecules, Springer-Verlag, Berlin 1995.

[7] V.L. Ermolaev, E.N. Bodunov, E.B. Sveshnikova, T.A. Shahverdov, Nonradiative Transfer of Electronic Excitation Energy Nauka, Leningrad 1977, Ch. 8 (in Russian).

[8] J.B. Birks, Photophysics of Aromatic Molecules, Wiley-Intersc., London 1969, p. 704.

[9] J.G. Calvert, J.N. Pitts, Jr., Photochemistry, J. Wiley and Sons, Inc. New York 1966.

[10] E.B. Sveshnikova, V.L. Ermolaev, Opt. Spectrosk. 30, 208 (1971).

[11] V.L. Ermolaev, E.B. Sveshnikova, Chen. Phys. Lett. 23, 349 (1973).

[12] B.R. Henry, W. Siebrand, J. Chem. Phys. 54, 1072 (1971).

[13] V.L. Ermolaev, E.B. Sveshnikova, E.N. Bodunov, Phys.-Usp. 39, 261 (1996) (very bad translation from Russian journal into English).

[14] S.H. Lin in: Radiationless Transitions, Ed. S.H. Lin, Academic Press, New York 1980, p. 363.

[15] E.B. Sveshnikova, S.P. Naumov, Opt. Spectrosk. 47, 279 (1979).

[16] G. Orlandi, W. Siebrand, Chem. Phys. Lett. 80, 399 (1981).

[17] M. Zander, Z. Naturforschung A 43, 393 (1988).

[18] E.B. Sveshnikova, V.P. Kondakova, Opt. Spectrosk. 50, 477 (1981).

[19] N.C. Yang, S.L. Murov, T.-C. Shieh, Chem. Phys. Lett. 3, 6 (1969).

[20] J.D. Simpson, H.W. Offen, J.G. Burr, Chem. Phys. Lett. 2, 383 (1968).

[21] R.J. Watts, S.J. Strickler, J. Chem. Phys. 49, 3867 (1968).

[22] T.D. Gierke, R.J. Watts, S.J. Strickler, J. Chem. Phys. 50, 5425 (1969).

[23] S.H. Lin, R. Bersohn, J. Chem. Phys. 48, 2732 (1968).

[24] J.-Ph. Grivet, Chem. Phys. Lett. 4, 104 (1969).

[25] B.R. Henry, J.L. Charlton, J. Amer. Chem. Soc. 95, 2782 (1973).

[26] A.J. Gordon, R.A. Ford, The Chemist's Companion. A Handbook of Practical Data, Techniques, and References, Wiley-Intersc., New York 1972, Ch. 4, Sec. VIII. 